\title{
Structure of Electrorheological Fluids under an Electric Field and a Shear Flow: Experiment and Computer Simulation
}

\author{
J. G. Cao, J. P. Huang,* and L. W. Zhou \\ Surface Physics Laboratory (National Key Laboratory) and Department of Physics, Fudan University, \\ Shanghai 200433, China
}

Received: February 24, 2006; In Final Form: April 14, 2006

\begin{abstract}
It is known that macroscopic properties of colloidal suspensions are often determined by the microstructure of the particles in the suspensions, depending on the interparticle, Brownian, and hydrodynamic (if any) forces. We take electrorheological (ER) fluids as an example. By using a computer simulation and an experimental approach, we investigate the structure of ER fluids subjected to both an electric field and a shear flow. The microstructure evolution from random structure, to chains, and then to stable lamellar patterns, observed in the experiments, agrees very well with that obtained in the simulations. It is shown that the formation of such lamellar patterns originates from the difference between the dipole moment induced in the particles suspended in the ER fluids without shear and the one with shear. The results on the relaxation process of structural formation and the internal structure of layers are also presented. Thus, it seems possible to achieve various structures and hence desired macroscopic properties of colloidal suspensions by adjusting external fields and, simultaneously, a shear flow.
\end{abstract}

\section{Introduction}

Colloidal suspensions can be found in many application areas, ${ }^{1}$ e.g., paints, inks, pharmaceuticals, food, and brakes. Colloid science and technology plays an important role in some emerging technologies, ranging from photonic crystals ${ }^{2,3}$ to microfluidics. ${ }^{4}$ The macroscopic properties of colloidal suspensions are determined by the microstructure of the particles suspended in the system. ${ }^{5}$ In the absence of shear, a wide range of stable microstructure in colloidal suspensions can be available in different materials, depending on interparticle forces and Brownian forces. In the presence of shear, the microstructure may depend on the strength of the shear, and it rearranges to accommodate the applied hydrodynamic forces (arising from the shear) and the interparticle and Brownian forces. When the suspension is subjected to a shear, the microstructure will affect the rheological properties accordingly. Hence, the intimate coupling between the shear and the microstructure during flow should be understood clearly, which is the main object of this work. The relevance lies in the central role of the shear-affected microstructure in determining the macroscopic properties of the suspensions and understanding and controlling the processing behavior of the suspensions with desired microstructures. The competition of different kinds of forces in colloid or complex fluid systems made them present variable patterns. Those patterns also provide a way to study the complex relation between the forces. For example, pattern formation has been observed and studied in magnetorheolegical (MR) ${ }^{6}$ or electrorheological (ER) suspensions. ${ }^{7}$ Different patterns correspond to their different rheological properties. ${ }^{8}$ In this paper, we shall focus on ER fluids only. Our results will also hold for other suspensions such as magnetorheological suspensions, ${ }^{9}$ ferrofluids, ${ }^{10,11}$ and electromagnetorheological fluids. ${ }^{12,13}$

An ER fluid ${ }^{14-17}$ is composed of nano- or micro-sized dielectric particles and insulating liquid. Under an external

* Corresponding author. E-mail: jphuang@fudan.edu.cn. electric field, the ER particles form chain and column structures, and their ground-state structure is the body-centered tetragonal lattice. The behavior of ER fluids subjected to both an electric field and a shear flow was investigated in the past decade ${ }^{7,18}$ lamellar structures were observed in ER fluids in the presence of oscillating shear flow and also predicated by numerical simulations in steady shear flows. ${ }^{19}$ The relations among chains, lamellar, and shear stress also been studied by Filisko. ${ }^{8}$ In theory, Klingenberg ${ }^{18,19}$ used a "two-fluid continuum model" to prove stripe formation in sheared suspensions. In this work, we shall present both a computer simulation and an experiment to understand the structure of ER fluids under an electric field and simultaneously a shear flow.

The paper is organized as follows. In Section II, we conduct an experiment to study the structure of an ER fluid containing strontium carbonate $\left(\mathrm{SrCO}_{3}\right)$ particles suspended in a silicon oi, under three conditions, namely, with neither fields nor shears, with fields only, and with both fields and shears. In Section III, we perform computer simulations to investigate the structure of an ER fluid under the three conditions. This is followed by Section IV, in which the results obtained from the simulations and experiment are presented. The paper ends with a discussion and conclusion in Section V.

\section{Experiment}

The ER fluid used in this experiment is made of strontium carbonate $\left(\mathrm{SrCO}_{3}\right)$ in a silicon oil. The particle diameter ranges from $0.5-1.0 \mu \mathrm{m}$. The relative dielectric constant of the particles is 800 (at $20 \mathrm{~Hz}$ ) and 300 (at $1 \mathrm{kHz}$ ), respectively, measured with a Hewlett-Packard 4284A precision LCR meter, and its conductivity is $10^{-6}(\Omega \mathrm{m})^{-1}$, measured with an EST121 digital ohmmeter for ultrahigh resistance. The kinetic viscosity of the silicon oil is $10 \mathrm{cSt}\left(\right.$ at $20^{\circ} \mathrm{C}$ ). The volume fraction is $1 \%$. First, the distribution of $\mathrm{SrCO}_{3}$ particles is random. When the external electric field $(E=1 \mathrm{kV} / \mathrm{mm})$ is applied, the $\mathrm{SrCO}_{3}$ particles form chains or columns. Under both an electric field 
$(E=1 \mathrm{kV} / \mathrm{mm})$ and a shear flow $\left(\dot{\gamma}=3 \mathrm{~s}^{-1}\right)$, the $\mathrm{SrCO}_{3}$ particles form lamellar structure. The response time of the structure is about several seconds.

\section{Computer Simulation}

A. Particle Interaction Force under Shear Flow. The ER effect originates from the induced interaction between the polarized particles in an ER fluid. Most theoretical models ${ }^{20-23}$ calculate the interactions between particles by assuming that the particles are at rest. However, in many applications, there is shear flow, which exerts force and torque on the particles, making them move and spin. It is found that the particles keep spinning even after they form particle chains. The above peculiar behavior of ER particles under shear flow determines the simulation assumption of an ER structure evolution under shear should different from before. The ER particle dipole moment changes in both direction and magnitude with shear rates. ${ }^{24}$ There should be a dipole moment tilted angle, which is the angle between the direction of the particle dipole moment and that of the electric field. Let us define it as:

$$
c \tan \phi=\frac{\left\langle m_{z}\right\rangle}{\left\langle m_{y}\right\rangle}=\frac{1}{\omega_{0} \tau}\left[\frac{P_{\infty}\left(1+\omega_{0}{ }^{2} \tau^{2}\right)}{P_{0}-P_{\infty}}+1\right]
$$

where $\left\langle m_{z}\right\rangle$ and $\left\langle m_{y}\right\rangle$ are the dipole moments of the particle in $z$ and $y$ direction, ${ }^{25}$ respectively, $\omega_{0}$ stands for the frequency of the particle spin, and $P_{0}$ represents the dipole moment of a dielectric sphere in a steady fluid (i.e., without shear or when shear rate is zero, $\dot{\gamma}=0) .{ }^{26}$ Analogously, in a fluid with an infinite shear rate, the dipole moment can be written as

$$
P_{\infty}=4 \pi R^{3} \epsilon_{\mathrm{f}} \frac{\epsilon_{\mathrm{p} \infty}-\epsilon_{\mathrm{f}}}{\epsilon_{\mathrm{p} \infty}+2 \epsilon_{\mathrm{f}}} E_{z}
$$

where $R$ represents the particle radius, $E_{\mathrm{z}}$ the external electrical field, and $\epsilon_{\mathrm{f}}$ the relative permittivity of the fluid. In eq $2, \epsilon_{\mathrm{p} \infty}$ is the particle's relative permittivity in a fluid with an infinite shear rate. In other words, $\epsilon_{\mathrm{p} \infty}$ means the high-frequency limit dielectric constant of the ER particles.

On the other hand, there will also be a decay factor of the dipole moment $k=\left\langle m_{y z}\right\rangle / P_{0}$, where $\left\langle m_{y z}\right\rangle$ is the total dipole moment defined as $\left\langle m_{y z}\right\rangle=\sqrt{\left\langle m_{y}\right\rangle^{2}+\left\langle m_{z}\right\rangle^{2}}$.

One may find out that the particle interaction force based on the modified Maxwell-Wagner model is ${ }^{24}$

$$
\begin{array}{r}
\mathbf{F}=\frac{3}{16} \pi \epsilon_{0} \epsilon_{\mathrm{f}} R^{2} \beta_{\mathrm{eff}}^{2} E_{\mathrm{loc}}{ }^{2}\left(\frac{R}{r_{i j}}\right)^{4}\left\{\left[3 \cos ^{2}(\theta-\phi)-1\right] \hat{\mathbf{r}}-\right. \\
\sin [2(\theta-\phi)] \hat{\theta}\}
\end{array}
$$

where $E_{\mathrm{loc}}$ is the local electric field, and $\beta_{\text {eff }}$ is the modified effective relative polarizability. Considering the decay factor $k, \beta_{\text {eff }}$ may be written as

$$
\beta_{\text {eff }}^{2}=k^{2} \beta_{\mathrm{d}} \frac{\left[(\omega \tau)^{2}+\frac{\beta_{\mathrm{c}}}{k \beta_{\mathrm{d}}}\right]^{2}+(\omega \tau)^{2}\left[1-\frac{\beta_{\mathrm{c}}}{k \beta_{\mathrm{d}}}\right]^{2}}{\left[1+(\omega \tau)^{2}\right]^{2}}
$$

where

$$
\beta_{\mathrm{d}}=\frac{\epsilon_{\mathrm{p}_{0}}-\epsilon_{\mathrm{f}}}{\epsilon_{\mathrm{p}_{0}}+2 \epsilon_{\mathrm{f}}} \beta_{\mathrm{c}}=\frac{\sigma_{\mathrm{p}}-\sigma_{\mathrm{f}}}{\sigma_{\mathrm{p}}+2 \sigma_{\mathrm{f}}}
$$

In eq $4, \omega$ denotes the frequency of the external electric field.
In eq $5, \sigma_{\mathrm{p}}$ stands for the conductivity of the ER particles, $\sigma_{\mathrm{f}}$ the conductivity of the host oil, and $\epsilon_{\mathrm{p}_{0}}$ the particle's relative permittivity in a fluid without shear. In fact, the relaxation time $\tau$ in eq 4 is quite different for materials with different conductivities, according to the Maxwell-Wagner theory of leaky dielectrics, because the relaxation process is originated from a finite conductivity of the particle and host medium. ${ }^{27}$ In the coexistence of electric and shear fields, by using meanfield arguments, the relaxation time is given as: ${ }^{28}$

$$
\tau=\tau_{\infty}+\frac{\tau_{0}-\tau_{\infty}}{\sqrt{1+\omega_{0}{ }^{2} \tau_{0}^{2}}}
$$

where

$$
\tau_{\infty}=\epsilon_{0}\left(\frac{\epsilon_{\mathrm{p} \infty}+2 \epsilon_{\mathrm{f}}}{\sigma_{\mathrm{p}}+2 \sigma_{\mathrm{f}}}\right), \quad \tau_{0}=\epsilon_{0}\left(\frac{\epsilon_{\mathrm{p}_{0}}+2 \epsilon_{\mathrm{f}}}{\sigma_{\mathrm{p}}+2 \sigma_{\mathrm{f}}}\right)
$$

When the shear rate is zero $(\dot{\gamma}=0)$, one has $k=1$ and $\phi=$ 0 , and the interparticle force expressed in the modified Maxwell-Wagner model reduces to that of the original Maxwell-Wagner model. ${ }^{24}$

B. Simulation Model. The ER system simulated in this paper consists of $N=600$ interacting spherical particles of dielectric constant $\epsilon_{\mathrm{p}}\left(\epsilon_{\mathrm{p}}=\epsilon_{\mathrm{p} \infty}\right.$ for $\dot{\gamma}=\infty ; \epsilon_{\mathrm{p}}=\epsilon_{p 0}$ for $\left.\dot{\gamma}=0\right)$ and radius $R$, suspended in a fluid medium of dielectric constant $\epsilon_{\mathrm{f}}$ with $\epsilon_{\mathrm{f}}$ $<\epsilon_{\mathrm{p}}$ and viscosity $\eta_{\mathrm{f}}$. The simulation box has a volume $V=L_{x}$ $\times L_{y} \times L_{z}$. An external electric field is applied along the $z$ direction. The particles are assumed to interact with each other via short-range repulsive and point-dipole forces, and to be affected by the solvent through Stokes drag (hydrodynamic interaction). A constant shear strain with a shear rate $\dot{\gamma}=3 \mathrm{~s}^{-1}$ is also imposed along the $y$ direction with a velocity field linearly varying along the $z$ direction. We assume that the buoyancy neutralizes the gravity. Following Guo et al. ${ }^{29}$ the forces exerted on each particle include: (1) electric dipole forces, (2) hardcore repulsive forces due to the neighboring particles, (3) the Stokes drag force arising from the host fluid, and (4) Brownian force $N_{i}(t)$, which is determined independently by a normal distribution with $\left\langle N_{i, \alpha}\right\rangle=0$ and $\left\langle N_{i, \alpha}(0) N_{i, \beta}(t)\right\rangle=6 \pi k_{\mathrm{B}} T \sigma \eta \delta_{\alpha \beta} \delta_{t}$, where $k_{\mathrm{B}}$ is the Boltzmann constant and $T$ the temperature. The forces acting on the $i$ th particle are

$$
\mathbf{F}_{i}=\sum_{j \neq i}\left(\mathbf{F}_{i, j}^{\mathrm{d}}+\mathbf{F}_{i, j}^{\mathrm{rep}}\right)+\sum_{j} \mathbf{F}_{i, j}^{\mathrm{d}, \mathrm{im}}+\mathbf{F}_{i}^{\mathrm{wall}}
$$

The dipolar force $\mathbf{F}_{i, j}^{\mathrm{d}}$ acting on the $i$ th particle at $r_{i}$ due to the $j$ th particle at $r_{j}$ is given by eq 3 . To simulate the hard spheres and the hard sphere-hard wall interaction, we introduce an exponential short-range repulsive force between the particles $i$ and $j$,

$$
\mathbf{F}_{i, j}^{\mathrm{rep}}=\left[3 P_{0}^{2} /\left(\epsilon_{\mathrm{f}} r_{i, j}{ }^{4}\right)\right] e^{-100\left(r_{i, j} / \mathrm{R}-1\right)} \hat{\mathbf{r}}
$$

and between particle $i$ and the two electrodes

$$
\mathbf{F}_{i, j}^{\text {wall }}=\left[3 P_{0}{ }^{2} /\left(\epsilon_{\mathrm{f}} r_{i, j}{ }^{4}\right)\right] e^{-100\left(z_{i} / \mathrm{R}-0.5\right)} \hat{\mathbf{z}}-F_{0} e^{-100\left(L_{z}-z_{i} / \mathrm{R}-0.5\right)} \hat{\mathbf{z}}
$$

where $r_{i, j}$ denotes the distance between the particles $i$ and $j$, and $L_{z}$ is the distance between two electrodes. In eq $8, F_{i, j}^{\mathrm{d} \text {,im }}$ is the summation force on the particle $i$ due to an infinite number of images of particle $j$ reflected about the electrodes. From the above discussion, we know the polarization direction is changed 

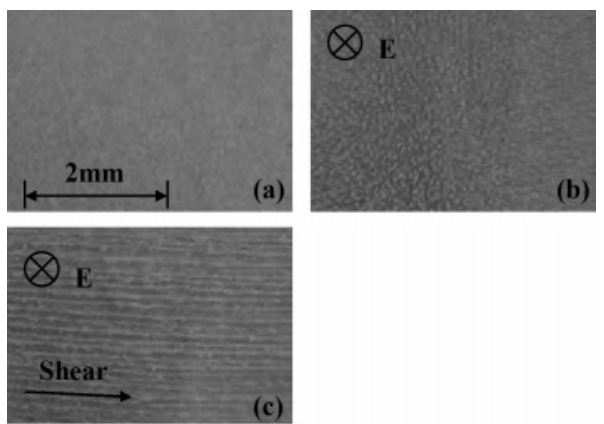

Figure 1. Experiment. Structural pattern of the $\mathrm{SrCO}_{3}$ particles suspended in the ER fluids under the three conditions: (a) with neither electric fields nor shear flows, (b) with electric fields only, and (c) with both electric fields and shear flows.

by shear. It made the images have different polarization. So the $F_{i, j}^{\mathrm{d}, \mathrm{im}}$ is given by:

$$
\begin{array}{r}
\mathbf{F}_{i, j}^{\mathrm{d}, \mathrm{im}}=\frac{3}{16} \pi \epsilon_{0} \epsilon_{\mathrm{f}} R^{2} \beta_{\mathrm{eff}}^{2} E_{\mathrm{loc}}{ }^{2}\left(\frac{R}{r_{i j}}\right)^{4}\{[\cos 2 \phi-3 \cos (2 \phi+ \\
\theta) \cos \theta] \hat{\mathbf{r}}-\sin (2 \theta+2 \phi) \hat{\theta}\}
\end{array}
$$

where $\phi$ denotes the angle as determined in eq 1 . According to

(a)

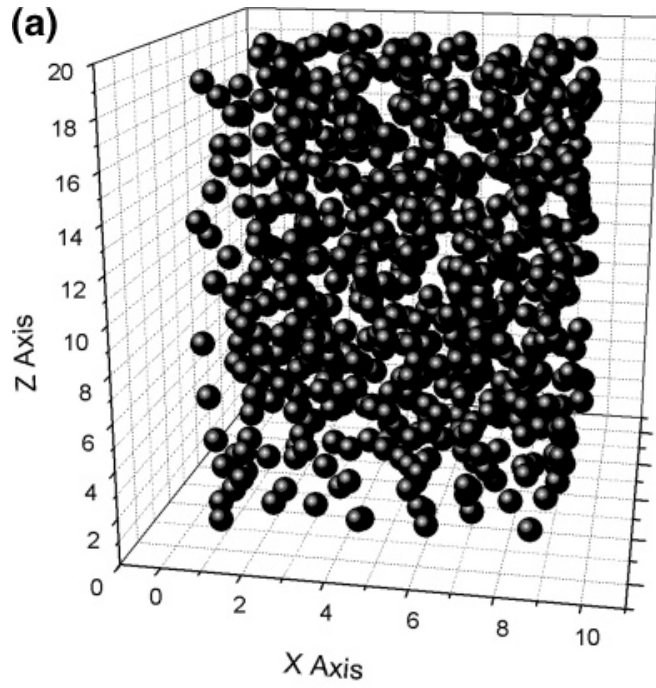

this equation, it can also be shown that the interaction force is caused to be lower than that of the static system.

The motion of $i$ th particle having mass $m$ at time $t$ and position $\mathbf{r}_{i}=x_{i} \hat{\mathbf{x}}+y_{i} \hat{\mathbf{y}}+z_{i} \hat{\mathbf{z}}$ is described by the following classical equation of motion:

$$
m \frac{\mathrm{d}^{2} \mathbf{r}_{i}}{\mathrm{~d} t^{2}}=\mathbf{F}_{i}-3 \pi R \eta_{\mathrm{f}}\left(\frac{\mathbf{r}_{i}}{t}-\dot{r} z_{i} \hat{\mathbf{y}}\right)+\mathbf{N}_{i}
$$

We introduce the following scaling factors for the electric force, Brownian force, length, and time, respectively: $F_{i}^{*}=$ $F_{i} /\left(P_{0}^{2} / \epsilon_{\mathrm{f}} R^{4}\right), N_{i}^{*}=N_{i} /\left(k_{\mathrm{B}} T / R\right), r^{*}=r / R$, and $t^{*}=t /\left(3 \pi \eta_{\mathrm{f}} R^{3 /}\right.$ $k_{\mathrm{B}} T$ ). By using these, eq 12 can be rewritten in the dimensionless form as

$$
A \frac{\mathrm{d}^{2} \mathbf{r}_{i}^{*}}{\mathrm{~d} t^{*^{2}}}=Q \mathbf{F}_{i}^{*}-\frac{\mathrm{d} \mathbf{r}_{i}^{*}}{\mathrm{~d} t^{*}}+8 P_{\mathrm{e}} z_{i}^{*} \hat{\mathbf{y}}+\mathbf{N}_{\mathbf{i}}^{*}
$$

For most real parameters of ER fluids, the magnitude $A=m k_{\mathrm{B}} T /$ $9 \pi^{2} \eta_{\mathrm{f}}^{2} R^{4}$ in eq 13 is very small, so in the following simulations,

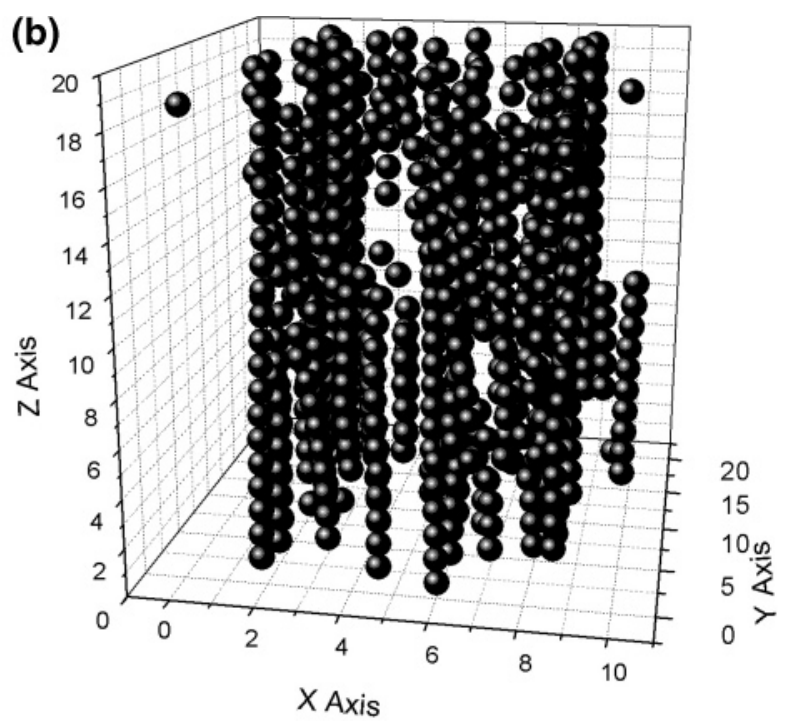

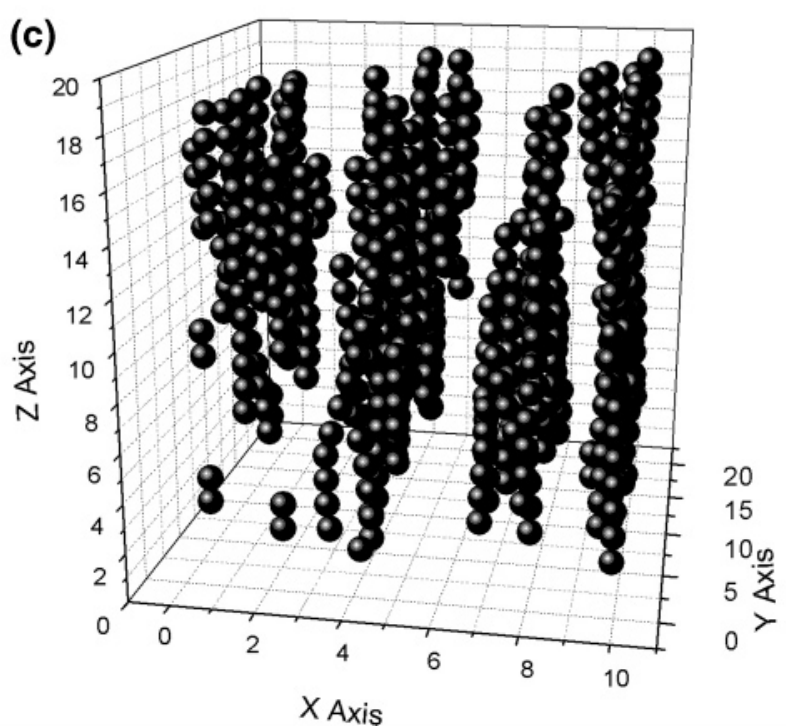

Figure 2. Computer simulation. Structural pattern of the particles suspended in the ER fluids under the three conditions as already listed in Figure 1. The electric field $(E=1 \mathrm{kV} / \mathrm{mm})$ in (b) and (c) is along the $z$ axis, and the shear $\left(\dot{\gamma}=3 \mathrm{~s}^{-1}\right)$ in (c) is along the $y$ axis. 


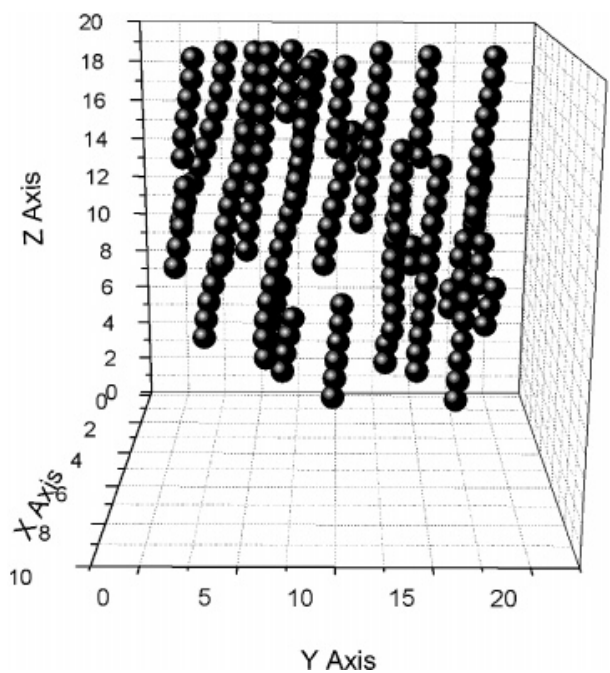

Figure 3. The internal structure of the ultraleft layer shown in Figure 2c.

we neglect this inertial effect. Thus, it is simplified as

$$
\frac{\mathrm{d} \mathbf{r}_{i}^{*}}{\mathrm{~d} t^{*}}=Q \mathbf{F}_{i}^{*}+8 P e z_{i}^{*} \hat{\mathbf{y}}+\mathbf{N}_{i}^{*}
$$

Now, we have two dimensionless parameters, $Q=P_{0}^{2} /$ $\left(\epsilon_{\mathrm{f}} R^{3} k_{\mathrm{B}} T\right)$ and $P_{\mathrm{e}}=3 \pi \eta_{\mathrm{f}} R^{3} \dot{\gamma} /\left(8 k_{\mathrm{B}} T\right)$, where $Q$ is the ratio of the dipolar force to the Brownian force, and $P_{\mathrm{e}}$ represents the ratio of the shear force to the Brownian force, also called Peclet number.

\section{Results}

Our simulations have been done for the parameters: $E_{0}=1$ $\mathrm{kV} / \mathrm{mm}, T=300 \mathrm{~K}, \epsilon_{\mathrm{p} 0}=800, \epsilon_{\mathrm{p} \infty}=300, \sigma=1.0 \times 10^{-6}$ $(\Omega \mathrm{m})^{-1}, \epsilon_{\mathrm{f}}=2$, and $\eta=0.01 \mathrm{pa}$. The shear rate $\dot{\gamma}$ is $3 \mathrm{~s}^{-1}$.

Figure 1 shows the experimental observation of the structure pattern of the $\mathrm{SrCO}_{3}$ particles suspended in the ER fluids under different conditions: (a) with neither electric fields nor shear flows, (b) with electric fields only, and (c) with both electric fields and shear flows. Figure 1a shows that the particles are randomly distributed in the suspension. From Figure $1 \mathrm{~b}$, we find that particle chains are formed, which is induced by the external applied electric field. In the presence of both an electric field and a shear, the lamellar structure was observed, as shown in Figure 1c. Such observations agree very well with the patterns obtains from our computer simulations, see Figure 2 where random microstructure, chain formation, and lamellar structure are shown in Figure 2a, b, and c, respectively. On the basis of our simulations, we found that the particles that have a very small decrease in the dipolar moment magnitude and title angle can form lamellar structure very well. The good comparison between experimental observations (Figure 1) and simulation patterns (Figure 2) also shows the fact that the physics presented in Section III is reasonable for understanding the formation of lamellar structure in real situations.

For more details, let us analyze the structure of each layer by the following three order parameters:

$$
C_{j}=\left|\frac{1}{N} \sum_{i=1}^{N} \exp \left(i \mathbf{b}_{\mathbf{j}} \cdot \mathbf{r}_{\mathbf{i}}\right)\right|
$$

where $j=1,2,3$, and the three reciprocal lattice vectors of $\mathbf{b}_{\mathbf{j}}$

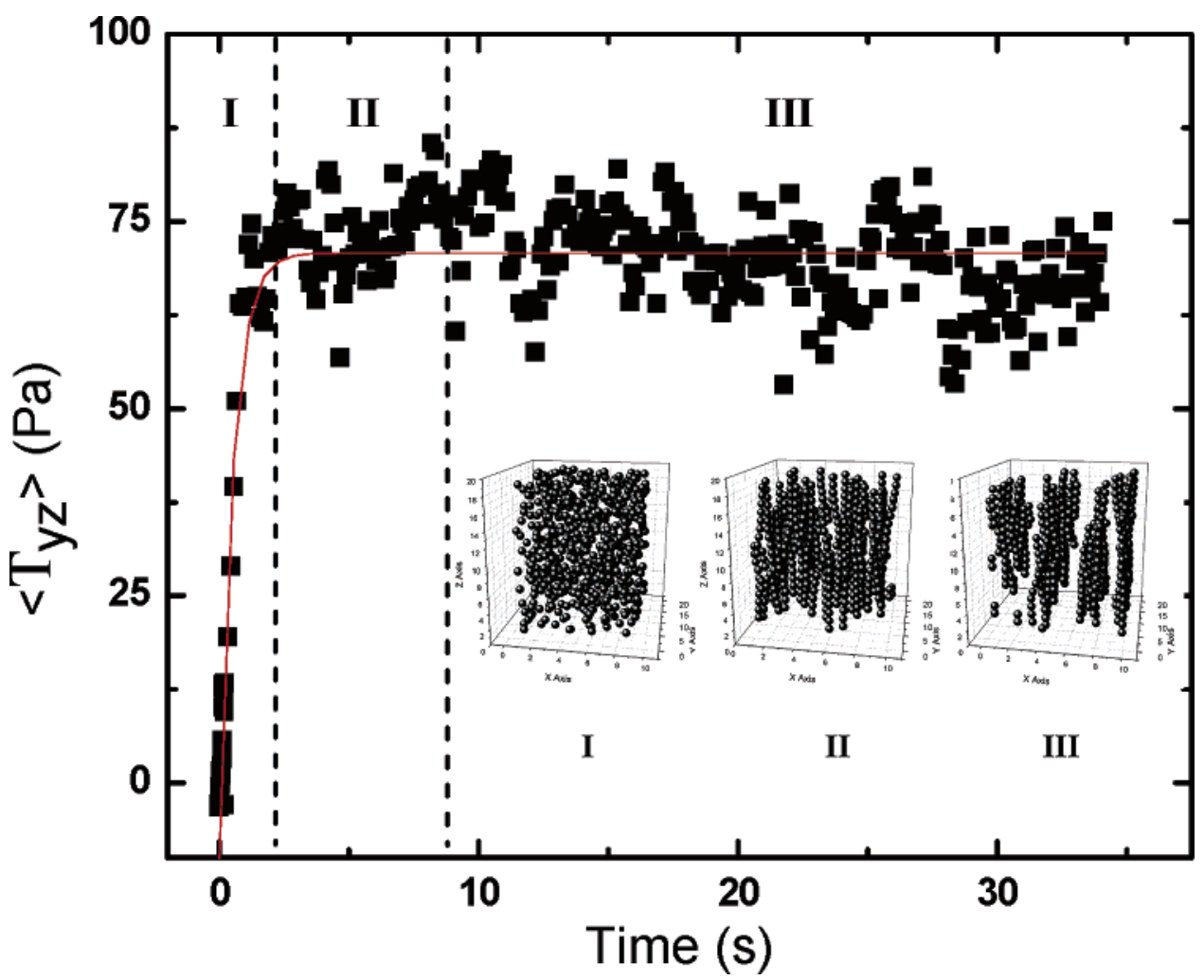

Figure 4. When both an electric field and a shear are simultaneously applied, the shear stress during the whole simulation period contains three steps (relaxation process): (I) The stress increases sharply but the distribution of the ER particles is still random for $2 \mathrm{~s}$ or so. (II) The stress tends to be stable for additional $7 \mathrm{~s}$ or so, while the lamellar pattern has not yet appeared. (III) After $9 \mathrm{~s}$ or so, the stress tends to stable and the lamellar structure appears. It is worth noting that the time displayed in this figure denotes the real time rather than the simulation time. The solid line in the figure is a guide for the eye. 
represent some known structures such as body-centered cubic, face-centered cubic, body-centered tetragonal, and hexagonal close-packed lattices. In these order parameters, $C_{3}$ characterizes the formation of chains along the $z$ direction, while $C_{1}$ and $C_{2}$ characterize the structure of particle arrangement in the $x y$ plane. To compare the $C_{j}$ for different structures, we can know the microstructure in each directions. Interestingly, the current simulation results show that the ultraleft layer displayed in Figure $2 \mathrm{c}$ is composed with tilted chains, see Figure 3. This actually holds for all the layers in the layer pattern.

In the dynamic state, reheological properties can be indicated by the effective viscosity $\eta_{\text {eff. }}$ This is calculated from the stress $T_{y z}$ of the particle interaction, $\eta_{\text {eff }}=\left\langle T_{y z} / \dot{\gamma}\right\rangle$, where $\left\langle T_{y z}\right\rangle$ is the $y z$-component of the stress tensor averaged over the simulation runs: 29

$$
\left\langle T_{y z}\right\rangle=\left\langle\frac{-1}{V} \sum_{i=1}^{N}\left(r_{i}\right)_{y}\left(F_{i}\right)_{z}\right\rangle
$$

In Figure 4, the relation between the stress and structure evolution is displayed for the case that both an electric field and a shear are applied simultaneously. In fact, Figure 4 shows the relaxation process corresponding to this case. Once the electric field and shear flow are applied, the stress increases sharply during two seconds or so (step I), then it tends to be stable for additional seven seconds or so with the appearance of chains, but without a lamellar pattern (step II). After nine seconds or so, the lamellar structure of the ER fluid becomes stable as the stress also tends to stable (step III). In the meantime, as the time goes, the structure changes from random structure (step I), to chains (step II), and then to a lamellar pattern (step III).

\section{Discussion and Conclusion}

Here, some comments are in order. It is known that macroscopic properties of colloidal suspensions are often determined by the microstructure of the particles in the suspensions, depending on the interparticle, Brownian, and hydrodynamic (if any) forces. In this paper, we have performed both computer simulations and experiment techniques to investigate the structure of ER fluids under different conditions. The ER fluids under consideration are monodispersed throughout the work. It is also interesting to see what happens for the structure if one includes the effect of polydispersity ${ }^{30,31}$ that may arise from fabrication and exist in real colloidal suspensions. For simplification, in simulations, one may first consider a bidisperse model. For a polydisperse model, one may assume the particle distribution to obey a log-normal distribution.

To sum up, by using a computer simulation and an experimental approach, we have investigated the structure evolution of ER fluids subjected to neither electric fields nor shears, an electric field only, and both an electric field and a shear flow. The pattern evolution, from random structure, to chains, and then to stable lamellar patterns, obtained from our computer simulations, agrees very well with our experimental observations. It has been shown that the formation of such lamellar pattern originates from the difference between the dipole moment induced in the particles suspended in the ER fluids without shear and the one in the particles with shear. Thus, it is possible to achieve different patterns or microstructure and hence desired macroscopic properties of colloidal suspensions like ER fluids, magnetorheological suspensions, ferrofluids, and electromagnetorheological fluids, by applying external fields and simultaneously a shear flow.

Acknowledgment. We acknowledge the financial support by the National Natural Science Foundation of China under grant nos. 1024402 and 10574027, and 10321003. J.G.C. acknowledges the financial support by the Fudan University Graduate Student Creative Foundation. J.P.H. acknowledges the financial support by the Shanghai Education Committee and the Shanghai Education Development Foundation ("Shu Guang" project) under grant no. KBH1512203, by the Scientific Research Foundation for the Returned Overseas Chinese Scholars, State Education Ministry, China, by Fudan University, and by the Jiangsu Key Laboratory of Thin Films, Suzhou University, China.

\section{References and Notes}

(1) Hiemenz, P. C.; Rajagopalan, R. Principles of Colloid and Surface Chemistry, 3rd ed.; Dekker: New York, 1997.

(2) Vlasov, Y. A.; Yao, N.; Norris, D. J. Adv. Mater. 1999, 11, 165.

(3) Tao R.; Xiao, D. Appl. Phys. Lett. 2002, 80, 4702.

(4) Terray, A.; Oakey, J.; Marr, D. W. M. Science 2002, 296, 1841.

(5) Larson, R. G. Rheology and Structure of Complex Fluids; Oxford University Press: Oxford, 1999.

(6) Cutillas, S.; Bossis, G.; Cebers, A. Phys. Rev. E 1998 57, 804

(7) Sun, J. M.; Tao, R. Phys. Rev. E, 1996, 53, 3732.

(8) Henley S.; Filisko, F. E. Int. J. Mod. Phys. B 2002, 16, 2286.

(9) Zhou, L.; Wen, W.; Sheng, P. Phys. Rev. Lett. 1998, 81, 1509.

(10) Odenbach, S. Magnetoviscous Effects in Ferrofluids; Springer: Berlin, 2002.

(11) Huang, J. P. J. Phys. Chem. B 2004, 108, 13901.

(12) Tao R.; Jiang, Q. Phys. Rev. E 1998, 57, 5761.

(13) Wen, W.; Wang, N.; Ma, H.; Lin, Z.; Tam, W. Y.; Chan, C. T.; Sheng, P. Phys. Rev. Lett. 1999, 82, 4248.

(14) Halsey, T. C. Science 1992, 258, 761.

(15) Goodwin, J. W.; Markham, G. M.; Vincent, B. J. Phys. Chem. B 1997, 101, 1961.

(16) Wen, W. J.; Huang, X. X.; Yang, S. H.; Lu, K. Q.; Sheng, P. Nature Mater. 2003, 2, 727.

(17) Huang, J. P. J. Phys. Chem. B 2005, 109, 4824

(18) Klingenberg, D. J.; Zukoski, C. F., IV. Langmuir 1990, 6, 15.

(19) von Pfeil, K.; Graham, M. D.; Klingenberg, D. J. Phys. Rev. Lett. 2002, 88, 188301.

(20) Klingenberg, D. J.; van Swol, F.; Zukoski, C. F. J. Chem. Phys. 1989, 91, 7888 .

(21) Khusid, B.; Acrivos, A. Phys. Rev. E 1995, 52, 1669

(22) Klingenberg, D. J.; van Swol, F.; Zukoski, C. F. J. Chem. Phys. 1991, 94, 6170

(23) Siu, Y. L.; Wan, J. T. K.; Yu, K. W. Phys. Rev. E 2001, 64, 051506.

(24) Cao, J. G.; Wang, J.; Zhou, L. W. Chem. Phys. Lett. 2006, 419, 149.

(25) Block, H.; Kluk, E.; Mcconnell, J.; Scaife, B. K. P. J. Colloid Interface Sci. 1984, 101, 320.

(26) Jackson, J. D. Classical Electrodynamics, 2nd ed.; Wiley: New York, 1975.

(27) Russel, W. B.; Saville, D. A.; Schowalter, W. R. Colloidal Dispersions; Cambridge University Press: Cambridge, U.K., 1989.

(28) Wan, J. T. K.; Yu, K. W.; Gu, G. Q. Phys. Rev. E 2001, 64, 061501

(29) Guo, H. X.; Mai, Z. H.; Tian, H. H. Phys. Rev. E 1996, 53, 3823.

(30) Petukhov, A. V.; van der Beek, D.; Dullens, R. P. A.; Dolbnya, I.

P.; Vroege, G. J.; Lekkerkerker, H. N. W. Phys. Rev. Lett. 2005, 95, 077801.

(31) Wilding, N. B.; Sollich, P.; Fasolo, M. Phys. Rev. Lett. 2005, 95, 155701. 